\title{
Human machine interface: robotizing the instinctive living
}

\begin{abstract}
The advancement in medical science and technology has revolutionized human interaction with machines, crafting one of the most promising state-of-the-art technological fields of today's world. This innovation has made doors opened to rehabilitation beyond patient assistance-Brain Computer Interface (BCI) has stepped forward towards life automation (brain controlled daily life actions), and entertainment (hands-free gaming). This paper reviews key techniques and sensors vital to this technological paramount, along with an appraisal to the commercially available sensors. An exclusive appraisal on state of-the-art BCI technologies has also been discussed conferring their application specific importance. The methodical study of these technologies and comparison of corresponding techniques and sensors is potentially useful to demonstrate the significance of brain machine interface.
\end{abstract}

Volume 4 Issue 5 - 2018

\author{
Ahmad Mahmood Tahir,' Jamshed lqbal, ${ }^{2}$ \\ Tauseef Aized ${ }^{3}$ \\ 'Department of Mechanical Engineering (DIME), PMAR Labs, \\ University of Genoa, Italy \\ ${ }^{2}$ Department of Electrical and Computer Engineering, University \\ of Jeddah, Saudi Arabia \\ ${ }^{3}$ University of Engineering and Technology, Pakistan
}

Correspondence: Ahmad Mahmood Tahir, Department of Mechanical Engineering (DIME), PMAR Labs, University of Genoa, Italy, Email tahir@dimec.unige.it

Received: April 25, 2018 | Published: September 21, 2018

\section{Introduction}

\section{The maiden companionship}

The recent state-of-the-art of brain computer interface has been revolutionized from a state which was presented by way of fiction just a few decades ago. The human brain electric signals were first recorded in 1929 by a German Scientist Hans Berger; ${ }^{1}$ however, it took 25 more years to realize the first brain-computer collaboration when Dr. W.G. Walter implemented slide projector progression in 1964. But the event of logging electric brain signals in 1929 may be acknowledged as anopening to the impending human lifestyle which Dr. W.G. Walter conceived as a brain controlled activity. Dr. Walter described it as Contingent Negative Variation (CNV) which represents the connotation and anticipation of human brain sensori motor signals in the form of electric signals. ${ }^{2}$ Till the end of last century, brain-computer interaction remained a laboratory activity confined to a small number of people working directly in this field as scientists and engineers, or to those who were associated subjectively in the experimentation. By the end of twentieth century, the research institutes and scientists involved in BCI research accomplished the recognition and taxonomy of major research goals aiming at basic and applied BCI research by focusingon technical issues and proceeding towards the development of standard research and assessment methodologies.

\section{The contemporary apprehension}

Currently there are more than a hundred research groups conducting research in this field around the world. ${ }^{1}$ In the fourth international BCI meeting held in 2010 at the Asilomar Conference Center in Monterey, California, USA, 260 participants represented 17 countries, ${ }^{4}$ In comparison with merely 50 participants from 7 countries in 1999 during the first meeting of the series. ${ }^{3}$ The major goal of BCI research is to develop state-of-the-art technologies to assist, augment or repair human's sensory motor or cognitive abilities. Modern BMIs implemented in the clinical studies as well as general experimentation are capable of refining and translating neuronal signals into motor instructions to reproduce arm and hand actuation in artificial actuators. ${ }^{5}$ Besides serving as an assistive technology for patients and handicapped people, $\mathrm{BCI}$ is being realized for rehabilitation purposes and instigated for entertainment in the form of hands-free gaming. ${ }^{4,6-8}$

\section{A brain computer interface $(\mathrm{BCI})$ outfit}

A BCI system generally consists of sensing electrodes, BCI transducers, control interface and output device controller. ${ }^{9}$ Some of the BCI systems may include HMI or control displays. Brain signals are transformed into electrical signals through electrodes. Brain signals can be logged-in from the scalp, brain surface, or from the neural activity of the brain. ${ }^{10}$ The BCI transducer comprises 'feature generator' and 'feature translator'. Some transducers may also employ 'artifact processor' to remove artifacts from the amplified electrical signal from electrodes. The 'feature generator' generates the neuro-mechanism using the amplified signals from brain. This neuro-mechanism is then translated into logical control signal through 'feature translator'. The 'interface controller' provides device control signals which are then physically carried out by 'device controller' engaging a device like a robotic arm, wheel chair, or a mobile robot etc. ${ }^{9,10}$ A general schematic of a real time human brain machine interface has been shown in Figure 1.

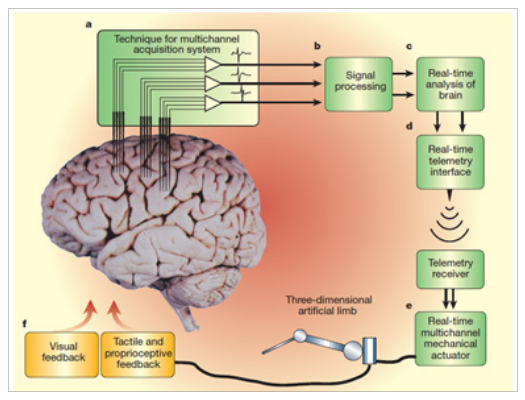

Figure I Human Brain Machine Interface General Organization. ${ }^{15}$ 


\section{$\mathrm{BCl}$ techniques and sensors}

The techniques that have been used to measure brain activity include Electroencephalography (EEG), Electrocorticogram (ECoG), Electromyography (EMG), Magneto-encephalography (MEG), Functional Magnetic Resonance Imaging (fMRI), Near-Infrared Spectroscopy (NIRS), Positron Emission Tomography (PEC), and Single Photon Emission Computed Tomography (SPECT). ${ }^{1,3,5,11-14}$ Sensors for BCI systems are categorized as invasive and non-invasive. In invasive systems, sensors are embedded into the brain through surgery. While non-invasive systems don't need any surgery and the sensors are mounted on the head-skin or on hair.

\section{Paper organization}

This paper provides an insight into the applied BCI techniques, sensors and an exclusive appraisal to recent developments in this research area. This paper is organized in 4 sections including first section about the Introduction, section II represents a comparative overview of BCI techniques; section III reviews various sensors for implementing a BCI system; a selective overview of the state-of theart is reported in Section IV; and finally, Section V comments on the conclusion and the future work.

\section{BCl technologies}

The major technologies for BCI systems that are being implemented in medical sciences, engineering and other relevant research areas include EEG, ECoG, EMG, MEG, fMRI, and NIRS. ${ }^{1,3,5,11-14}$ A brief account on these technologies is presented here.

EEG: Electroencephalography is a non-invasive technique. It detects brain activity through electrodes placed on scalp sensing brain neural activity. It is the most widely employed technology for BCI systems owing to its pacified interface technique. ${ }^{16}$ Electric signals are detectedfrom the scalp through electrodes and communicated to $\mathrm{A} / \mathrm{D}$ converter after amplification. The digital signal from ADC is then used for further required processing. EEG is a faster and cheaper method in comparison with other technologies. Its main shortcoming is that the exact location of the Region of Interest (ROI) in the selected brain portion should be known in order to collect some meaningful information. Typical EEG electrodes are shown in Figure 2 and an EEG setup is illustrated in Figure 3a. It has a module which monitors the brain signal patterns and gives meaningful information. Electrodes are placed on the skull using an EEG cap (Figure 3b), which ensures the required information from the brain. ${ }^{17}$

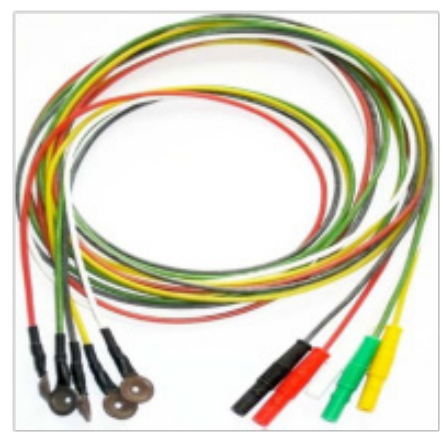

Figure 2 EEG electrodes.
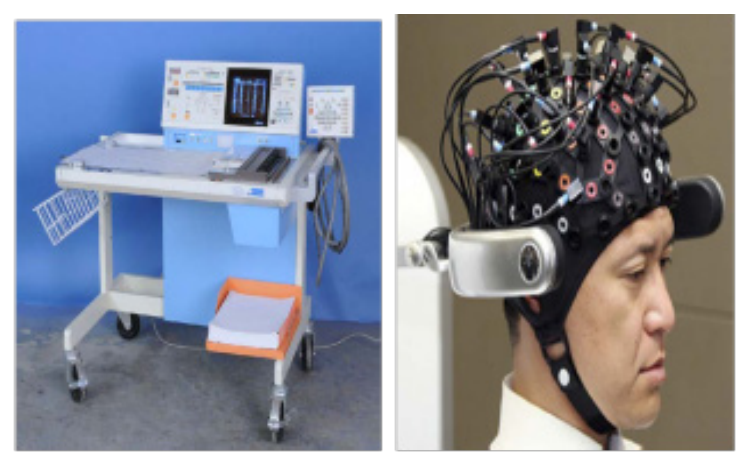

Figure 3 EEG: (a) A typical system (b) Helmet equipped with sensors.

ECoG: Electrocorticography is an invasive technique. This technique needs proper surgery of the brain to plant the sensors..$^{18}$ This method has been applied on animals like monkeys and rats. Research work reports the plantation of such sensors in humans as well. ECoG is mainly used to analyze the anatomy of animal's brain. Setup time is very less as compared to the other techniques. The main disadvantage in ECoG is the limited availability of resources.

ECG: Electrocardiography is a non invasive technique in which electrical activity of the heart is monitored. In this method, sensors are placed on the chest of the human/animal body to receive the heart activity, which is then measured by external equipment. ${ }^{19}$ In this technique, heart beat rhythm is estimated through ECG electrodes. The main disadvantage of this technique is that its operation is restricted to a single purpose i.e. heart. Experts in medical field can use this technique properly. ECG reads heart electrical conduction system: if the sensory stimulus is low in intensity then the motor effect of the neurons, which is in the form of electrical signals to the heart, will consequently low in intensity. In that case, ECG recordings will have peaks of small amplitude.

EMG: Electromyography is a noninvasive technique having sensors placed on the muscles of the body. An electromyography can also be used to measure electrical signals generated from the skeletal muscles. When an unusual action is happened in the muscles (e.g. biceps pump), EMG provides a proper feedback. ${ }^{20}$ This method has faster response as compared to EEG. EMG reads electrical signals from the muscles to interpret some meaningful information from the brain. If the pumping force of the muscle is low in intensity, then the electrical signal from the brain will also be in low amplitude mode.

MEG: Magneto Encephalography is a non invasive technique in which brain signals are recorded using magnetic fields, produced by the electric current which is naturally generated in the brain. ${ }^{3,21}$ Several research activitiesareon trackin this regard especially in the area of neuro feedback. This method requires apparatus to record minute brain signals whileprotecting them from stronger environmental magnetic fields.

fMRI: Functional Magnetic Resonance Imaging is a non invasive technique in which brain signals are collected through blood flow of a human. When there is a dissimilar hemodynamic behavior, brain signals will be detected through fMRI. In this method, high quality images are taken from different body parts for the analysis of the blood flow. Recent research is addressing the inter-relationship of EEG and fMRI. ${ }^{22}$ A typical machine for fMRI is shown in Figure 4. 


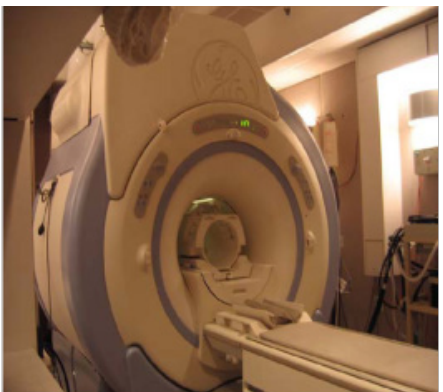

Figure $4 \mathrm{~A}$ typical machine for functional MRI.
NIRS: Near Infrared Spectroscopy is a noninvasive technique, which uses electromagnetic spectrum (ranges from $800 \mathrm{~nm}$ to $2500 \mathrm{~nm}$ ) to monitor electrical activity of blood and sugar level etc. This technique is being used in medical field as well as in other research areas such as robotics, food control etc. NIRS is very expensive technique as compared to other techniques. It is highly intricate to generate efficient control through NIRS since the signal processing methodologies are yet to be full-grown. ${ }^{23}$ Table 1 summarizes the features and comparative analysis of these technologies.

Table I summarizes the features and comparative analysis of these technologies

\begin{tabular}{|c|c|c|}
\hline Technology & Merits/Features & Limitations/ Research trends \\
\hline \multirow[t]{5}{*}{ EEG } & i. Non-invasive technique & \multirow{3}{*}{$\begin{array}{l}\text { i. Exact location of the brain portion is mandatory in } \\
\text { order to get meaningful brain signal. }\end{array}$} \\
\hline & ii. No brain surgery is required. & \\
\hline & iii. Faster and Cheaper than ECoG & \\
\hline & $\begin{array}{l}\text { iv. Used for analysis of all parts of the human body, animal } \\
\text { etc. }\end{array}$ & \multirow[t]{2}{*}{$\begin{array}{l}\text { ii. Use of different gel ointment on the head surface has } \\
\text { also negative implications. }\end{array}$} \\
\hline & v. $85 \% \mathrm{BCl}$ designs incorporate this technology & \\
\hline \multirow[t]{4}{*}{ ECoG } & i. Invasive technique & \multirow{2}{*}{$\begin{array}{l}\text { i. It is only applicable in animals as brain surgery is } \\
\text { required }\end{array}$} \\
\hline & ii. Better brain signal than EEG & \\
\hline & iii. Setup time is also less as compared to EEG & \multirow{2}{*}{$\begin{array}{l}\text { ii. Research on human implantation has recently been } \\
\text { started and limited resources are available for this } \\
\text { technique }\end{array}$} \\
\hline & $\begin{array}{l}\text { iv. A very good tool of analysis of cell structure and tissue } \\
\text { structures }\end{array}$ & \\
\hline \multirow[t]{3}{*}{ ECG } & i. Non-invasive technique & $\begin{array}{l}\text { i. Limited purpose: made only for single part of the body } \\
\text { (heart) }\end{array}$ \\
\hline & ii. Estimates the heat beat rhythm or monitors the unusual & \multirow{2}{*}{ ii. Special expertise is required for using ECG technology. } \\
\hline & iii. Heart beat is dependent on sensory stimulus. & \\
\hline \multirow[t]{3}{*}{ EMG } & i. Non-invasive technique & i. Expensive technique than EEG \\
\hline & ii. Used for muscular activities analysis. & ii. EMG based emotion control and neuro feedback using \\
\hline & iii. Faster than EEG & EMG are the current challenges. \\
\hline \multirow[t]{2}{*}{ MEG } & i. Non-invasive technique & $\begin{array}{l}\text { i.Very expensive technique as compared with all above } \\
\text { mentioned techniques }\end{array}$ \\
\hline & ii. Brain signals are collected through strong magnetic fields & $\begin{array}{l}\text { ii. Although widely used in medical but does not find much } \\
\text { presence in other research areas. }\end{array}$ \\
\hline \multirow[t]{2}{*}{ fMRI } & i. Non-invasive technique & $\begin{array}{l}\text { i. Expensive technique as compared to the EEG, but } \\
\text { cheaper than MEG. }\end{array}$ \\
\hline & $\begin{array}{l}\text { ii. High quality images are taken from human body parts, } \\
\text { which give an overview of blood flow in different body } \\
\text { parts. }\end{array}$ & $\begin{array}{l}\text { ii. fMRI \& EEG inter-relationship is a current challenging } \\
\text { research area. }\end{array}$ \\
\hline \multirow[t]{2}{*}{ NIRS } & i. Non-invasive technique & $\begin{array}{l}\text { i.Very expensive technique as compared to the other } \\
\text { techniques. }\end{array}$ \\
\hline & $\begin{array}{l}\text { ii. Electromagnetic spectrum is used to analyze blood and } \\
\text { sugar level in humans. }\end{array}$ & $\begin{array}{l}\text { ii. Current challenging research trends include } \\
\text { implementation of NIRS in robotics and food control. }\end{array}$ \\
\hline
\end{tabular}

\section{Review of sensors for $\mathrm{BCl}$}

There are different kinds of commercially available sensors for $\mathrm{BCI}$ corresponding to BCI types. Out of the two types of BCIs, ${ }^{24}$ Invasive BCI is related with the surgery. Proper plantation of sensors is required inside the brain to collect signals from the brain. ${ }^{25,26}$ Invasive procedures have two types of available sensors: Implantable and non- implantable micro electrode arrays (Figure 5). Non implantable electrodes are used in the dead species analysis e.g. cell structure and tissue structure. Micro-wire electrodes are used for this purpose. Triangulation method is applied in the course of the brain surgery to calculate the exact locations of neurons. On the contrary, Implantable electrodes are used in the analysis of living species according to the environmental changes in the brain. Implantable micro electrode arrays are of three types: Utah arrays, Michigan arrays and Flexible arrays. Utah arrays are silicon based electrodes. Brain signals are collected from the tip of each electrode. There are 100 needles in the electrode, which is the disadvantage of Utah arrays. Because of fixed parameters, information carried from the brain portion is very limited. Michigan arrays are also silicon based arrays. The major advantage of Michigan arrays is that there are no fixed parameters contrary to the Utah arrays, and these arrays allow higher compactness for 
implantation and spatial resolution as compared to the Utah arrays. Flexible arrays are advanced form of micro electrode arrays. They provide better match with specie skin to make better signal collection. All of these sensors are used in ECoG. This technique has been applied on animals like rats; monkey, pig etc.

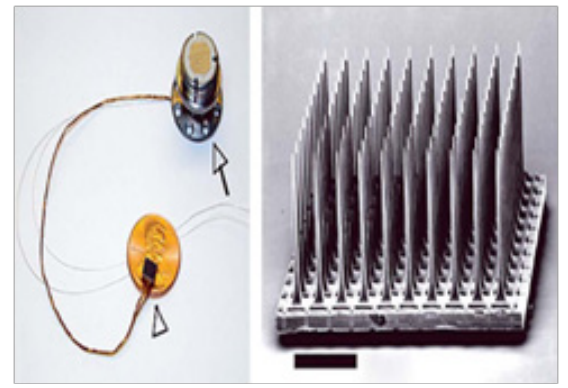

Figure $5 \mathrm{~A}$ Micro electrode arrays with a cable.

Local Field Potential (LFP) monitors the brain signals through non-invasive method. Brain surgery is not required in LFP. It has two types: capacitive (Figure 6) and non capacitive electrodes. Non capacitive electrodes have better performance ratio than the capacitive electrodes. Because of its material and chemical behavior, no effect is occurred when current passes through it. These sensors are widely used because of their low cost, and ease of use. 80 to $90 \%$ BCI designs use these sensors. Wet Electrodes Arrays (WEA) are also special purpose electrodes. These are also Ag/Agcl sensors. These are low impedance electrodes to achieve better brain signal. Special gel is used to attach electrodes on the head surface. Hairs need to be washed properly after the completion of the experiment. All of these sensors are used in EEG and MEG. Hybrid dry Electrode Sensor Array (HESA) is a great advancement in BCI. These electrodes perceive EEG signals through hair. No skin ointment or gel is required. The sensors consist of a set of pins that aresmart enough to stretch through hair without affecting the hair inside the pins. Bristle sensor is one of the main types of dry electrodes. These sensors have an amplifier circuit and a common mode rejecter for rejecting common mode signals in the input. It can record EEG signals for an unlimited time. These sensors have great sensitivity to high impedance, and resistive and capacitive behavior of the brain portion. These can be used in the light without affecting their performance. These sensors record brain signals through normal resistive electrodes and at the same time estimate the same signals using capacitive electrodes. Their Signal to Noise ratio (SNR) is better than wet electrodes. These are used in neuro-feedback research. Figure 7 \& Figure 8 shows hybrid bio-electrode transducers from Quasar.
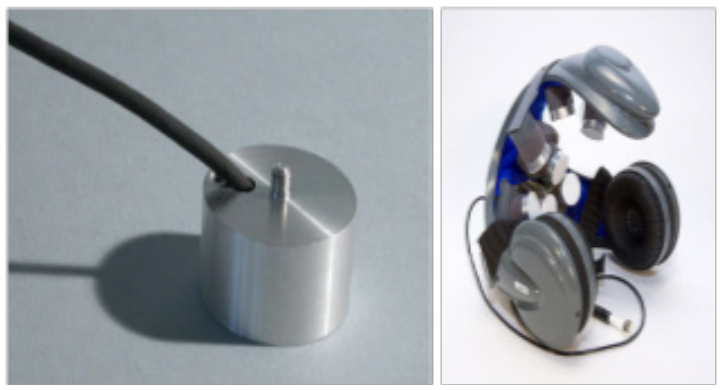

Figure 6 Capacitive electrode and its headset.

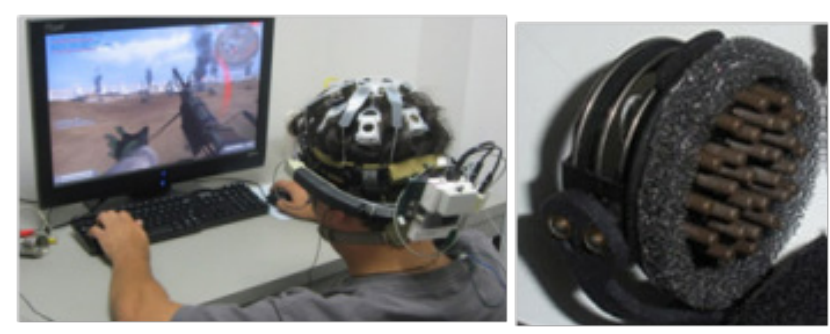

Figure 7 Hybrid Bio-electrode transducer by Quasar.

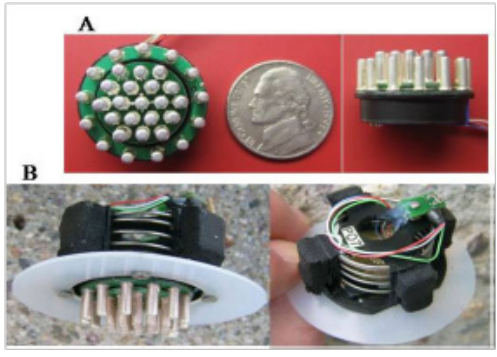

Figure 8 Quasar hybrid bio sensors with its internal circuitry.

Superconducting Quantum Interference Device (SQUID) Magnetometers are used in MEG. These magnetometers are used in recording brain signals, and exhibit better performance than EEG recording. The setup is huge in size and needs a special dedicated room. Electrodes need to be positioned inside magnetically shielded room. The main objective is to attain higher magnetic fields to achieve better brain signals recording. This technique, though used in medical field, has limited usage because of cost. MRI scanner equipment (Siemens trio scanner) is used for fMRI. It scans the whole body using large equipment and performs imaging of various parts of the body and estimates different hemodynamic behavior of the body parts. This technique is too expensive. In NIRS, the INVOS Cerebral/ Somatic Oximeter or sensor is used. It monitors the amount of blood and oxygen diffusion in the brain as well as in the body tissues to overcome their abnormal behavior. It is the only COTS available non-invasive oximeter. Manufacturing companies which are famous in designing BCI sensors include Quasar (US), Star Labs, G-Tec (Austria), Mindmedia (Netherlands) etc.

\section{State-of-the-art}

The fascination of the technology and the functional importance of BCI systems have steered high-tech developments. The first applied task that was achieved using this interface was the alphabet selection and the cursor control. ${ }^{27}$ This section presents some state-of-the-art in the field of BCI.

Remote-brained humanoid: Remote-brain is an approach for humanoids which primarily liberated such robots from an onboard brain. A remote brain makes the control of a humanoid more dynamic and endures structural stability avoiding a heavy on-body brain. This approach makes humanoids able to generate required motions more efficiently and effectively. ${ }^{28}$ One such system was developed at University of Tokyo in 1998 for a whole-body-action robot. That system eradicated a major shortcoming of previously developed systems. Older systems employed orthodox wireless connection for remote brain actions which restricted multiple actuator control. The new system 'Haru' (Figure 9) realized a flexible actuation control 
and multiple sensors organization employing a nervous system. It was a 22 DOF robot with 11 microprocessors which corresponds to the nervous control system of humans. The microprocessors network facilitated the collection of sensory information and distribute actuation signals locally for simultaneous multiple actuation control. For efficient execution of whole body actions, four control modes were implemented in addition to the $\mathrm{R} / \mathrm{C}$ servo method. Two-level control structure, joint level (lower control architecture) and joint coordination level (higher control architecture) was instigated in order to effectively use all of the control modes. The system was able to manipulate unknown objects and step on undefined paths. To execute required motions successfully, the humanoid was able to switch control modes adjusting the control parameters as some motions like knee movements need joints coordination.

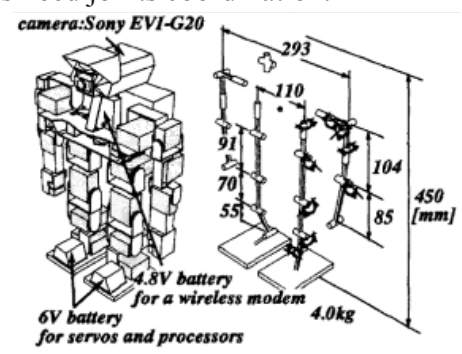

Figure 9 Haru - Remote-Brained Humanoid.

Electrode for sensory motor: A Ceramic Based Multi-Site (CBMS) electrode was developed by the Biomedical Engineering Department at Drexel University in 2001. The electrode was able to generate limb movements by producing motor neurons employing the spinal cord neurons. Reverse photolithography was used to pattern four recording sites, conducting lines and bonding pads on the ceramic substrate. The electrode was designed for prosthetic devices with a capability of recording across numerous brain regions, restoring sensory feedback by exciting neural tissues, and determining the localized concentration of neuromodulators. ${ }^{29}$ The working functionality of such a BCI system is depicted in Figure 10.

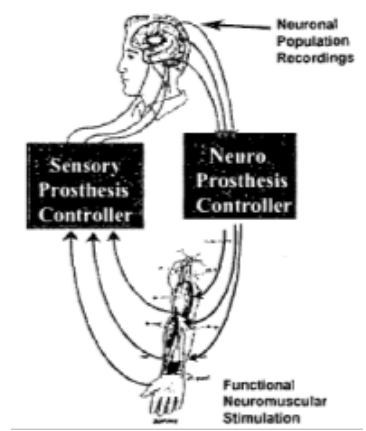

Figure $10 \mathrm{BCl}$ for Sensory and Motor Prosthetic Devices.

Continuous shared control system for BMI: A dexterous control scheme was realized by a group of scientists from MIT, University of California and The Duke University in 2004. The paradigm shared the control between the signals from a brain and a local sensors' network. The command signals from brain apprehended the pose of the end effector of a 3-DOF robot while the reactive signals from the local sensors aided surrounding information of the robot serving as robot reflexes. The control scheme instigated both operator control and the autonomy of the robot breeding an innovative control approach. ${ }^{30}$ Figure 11 illustrates a robotic gripper employing continuous shared control scheme. The reflexes of the robot assisted in avoiding gripper collisions modifying the command trajectory. Repulsive sensors on the outer sides of the gripper evaded sidewise impact. The gripper was able to hover over flat surfaces and fairly curved surfaces employing outer repulsive sensors and a central attractive sensor. While approaching an object and detecting within the gripping area, the central attractive sensor generated reflexive gripping making the command trajectory more instinctive. The best task performance for this BMI control technique was achieved with employing $70 \%$ command signals from the brain and 30\% reflexive signals from the local sensory array. Robot tele operation for patients: A BCI was developed in collaboration between the University of Palermo and the University of Padua, Italy in 2009. The system was developed for the patients with neuromuscular diseases providing a graphical user interface to manipulate a mobile robot through brain commands. ${ }^{31}$ Two beforehand robots were employed in that work: PeopleBot from University of Palermo and Pioneer3 from Georgia Tech. Figure 12 shows the robots and the GUI. People Bot is a mobile robot for effective indoor operations with an ability to manipulate objects through a gripper, transmit video images through a pan-tilt camera, and to follow colours. Pioneer3 with four drive wheels is able to work in outdoor terrains. BCI communication was done employing TCP-IP client server system. The user can operate the selected robot concentrating on the four arrows given on the GUI. This was an EEG based system recording and transforming motor intentions of the user. The system was tested successfully with wired, wireless and over the VPN without significant difference in the results. In the actual application of this system, the user was able to manipulate the robot in the museum of Agrigento, Italy through a GUI on San Camillo's computer in Venice, Italy. ${ }^{31}$

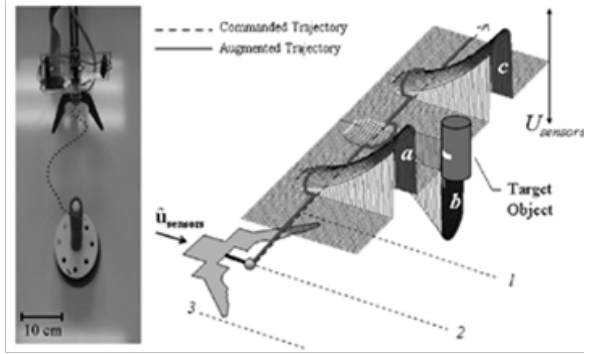

Figure II Robot gripper with CSC BMI.

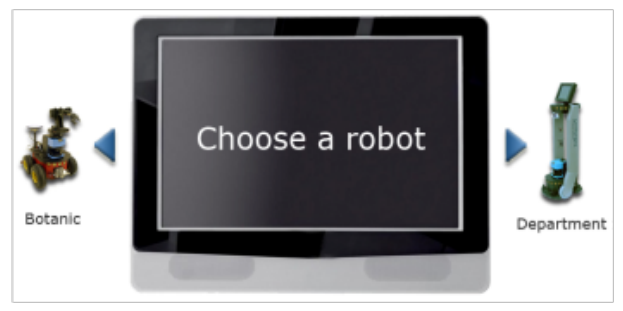

Figure 12 Robots and the GUI.

Motor imagery based BMI: An EEG based approach to control a robotic arm was comprehended by the experts from Maebashi Institute Japan, National University of Singapore and Santa Clara University USA. They employed different brain excitation degrees of humans to design four different tasks: idling state, gazing state, motion-imagery state, and motion and motion-imagery state. These four tasks (Figure 13) were accomplished by extracting mu and beta rhythms of EEG with short time FFT. A required control of the robotic arm was achieved experimentally with $100 \mathrm{~ms}$ control period..$^{32} \mathrm{~A}$ onejoint robot arm was controlled in this experiment. EEG signals to lift 
an arm was obtained and divided with shot-time FFT window to get the power spectra. The obtained magnitudes of mu and beta were implemented to achieve the featured movements.
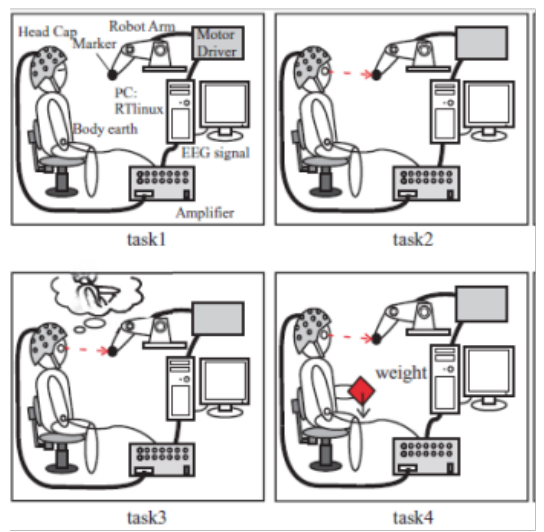

Figure I 3 Motion Imagery based BMI.

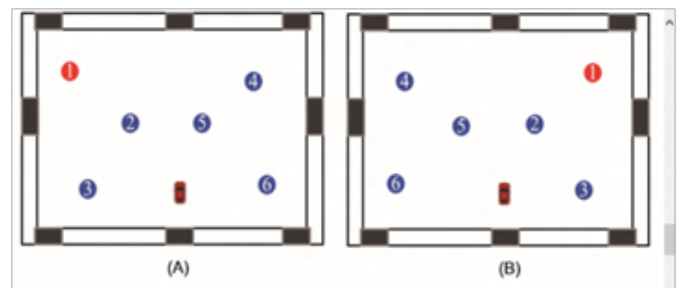

Figure I 4 The GUI for Wheelchair with pre-defined routs and flashing buttons for acceleration and deceleration.

BCI for wheelchair control: A hybrid BCI system employing motor-imagery and P300 was realized by Chinese scientists in 2011 to provide accurate multiple commands for multi-degree continuous control of a wheelchair. The system implemented four tasks-left and right direction control, and acceleration and deceleration of the wheelchair. Motor imagery was used to control the left right directions whereas the speed control was achieved through the hybrid mode. To accelerate, the user needs to govern his/her attention to the specific flashing button on the GUI (Figure 14). ${ }^{33}$ To decelerate, the user would imagine foot imagery and ignoring the flash buttons. So the system would be implementing one of the five EEG signals from the user which are left-hand motor imagery, right-hand motor imagery, foot motor imagery, flashing button attention, and idle. This BCI system successfully implemented the speed control accomplishing given tasks at high speed for $57.75 \mathrm{~s}$ and at low speed for $26.67 \mathrm{~s}$ during the trial. The hybrid control accuracy was found to be better than the motor-imagery control during the real world experiments for this hybrid BCI system ${ }^{33}$

Miscellaneous BCI systems: There are many other systems which have been realized in the field of BCI instigating various methodologies. Riccardo Poli, Mathew Salvaris, and Caterina Cinel proposed a mouse control $\mathrm{BCI}$ employing genetic programming which produced better performance results than Support Vector Machine technology for BCI ${ }^{29}$ Wei Li, Christian Jaramillo, Yunyi Li developed a Mind Control System for humanoids using 32 channel EEG and a CCD camera. Two robots KT-X PC with 20 DOFs and NAO H25 with 25 DOFs were used in experimentation to implement three brain activities including 'turning right', 'turning left' and 'walking forward' ${ }^{30}$ A graze-brain computer interface has been reported in $^{34}$ which is a cue-based interface that executes brain tasks using the motor-imagery. This BCI was successfully trialed comprehending a 'virtual keyboard', 'hand orthosis' operation, BCI training of a patient, and examining the limits of information transfer through this interface.

\section{Conclusion}

Scientists and researchers in the area of medical science and technology have been convinced that our brain is the hub and controller of Central Nervous System (CNS). Billions of neurons in the brain help the human to have self-control, plan the tasks, develop reasons and think in an abstract way. The efforts to have autonomous systems have emerged various inter-disciplinary research fields. Brain Computer Interface (BCI) is one such current hot area, which permits the human brain to directly communicate with an external system. Its main role is to assist, augment or repair human's sensory motor or cognitive abilities. A BCI system consists of various modules; sensing electrodes, signal acquisition and processing, features extraction and classification, pattern recognition, interface control. This paper reviews the state of-the-art of key techniques and sensors involved in $\mathrm{BCI}$ systems and present the results of this systematic and comparative study. The paper gives a detailed overview of associated techniques by discussing their performance scope and applications. In the field of medicine, several techniques have been proposed and implemented on $\mathrm{BCI}$ systems. These are based on ECOG, EEG, MEG, EMG, fMRI and NIRS. Sensors for these techniques can be broadly categorized into invasive and non-invasive type. In invasive BCI, surgery in the brain permits plantation of sensors into the brain thus generating electrical signals. In contrast, non-invasive BCI collects the electrical signals through sensors mounted on the head skin or hair. Invasive sensors are employed in ECOG technique and are usually composed up of microelectrode arrays. These are used in dead animals for analyzing their body structure (non-implanted) and in lively animals for their behavioral analysis (implanted). Recent approaches address human implantation as well. Michigan and Utah electrode arrays are examples of invasive BCI. Most of the other technologies (e.g. EEG, MEG, EMG, fMRI, NIRS) exploit the benefits of non-invasive sensors. In EEG, the types of electrodes used include local field electrodes, nonpolarizable electrodes, capacitive and non-capacitive electrodes, wet and dry electrodes. Different metals and non-metals are implanted in these electrodes and an electrochemical process is responsible for the flow of current. MEG, a very expensive technique, relies on magnetic field (Magnetometer) to capture the brain signals. EMG is a technique related with analysis and recording of skeletal muscles activities. The vital region of the brain can precisely be controlled using fMRI based BCI that works on the perception of blood circulation. The blood circulation is analyzed through the sensors. NIRS is a technique to estimate local cortical brain activities and offers portability, accessibility and safety. Our analysis of reported technologies and discussion on the available sensors are potentially useful for assessing impact of this interface and can serve as a guide for medical scientists working in this area.

\section{Acknowledgements}

None.

\section{Conflict of interest}

The author declares there is no conflict of interest. 


\section{References}

1. Graimann B, Allison B, Pfurtscheller G. Brain-computer interfaces: a gentle introduction. Brain-Computer Interfaces. Springer-Verlag Berlin Heidelberg; 2010:1-27.

2. Wieser HG. What are cognitive evoked potentials? Schweizer Archiv Für Neurologie Und Psychiatrie. 1998;149(6):268-272.

3. Wolpaw JR, Birbaumer N, Heetderks WJ, et al. Brain-computer interface technology: a review of the first international meeting. IEEE Trans Rehabil Eng. 2000;8(2):164-173.

4. Vaughan TM, Wolpaw JR. Editorial: special issue containing contributions from the fourth international brain-computer interface meeting. J Neural Eng. 2011;8(2):020201.

5. Lebedev MA, Nicolelis MAL. Brain-machine interfaces: past, present and future. Trends Neurosci. 2006;29(9):536-545.

6. Wang Q, Sourina O, Nguyen MK. EEG-based "serious" games design for medical applications. IEEE International Conference on Cyberworlds; 2010:270-276.

7. Tangermann MW, Krauledat M, Grzeska K, et al. Playing pinball with non-invasive BCI. 22nd Annual Conference on Neural Information Processing Systems; 2008.

8. Pires G, Torres M, Casaleiro N, et al. Playing tetris with non-invasive BCI. IEEE 1st International Conference on Serious Games and Applications for Health (SeGAH); 2011 Nov 16-18; Braga, Portugal. 2011.

9. Mason SG, GE Birch. A general framework for brain-computer interface design. IEEE Transactions On Neural Systems And Rehabilitation Engineering. 2003;11(1):70-85.

10. Bashashati M, Fatourechi RK, Ward RK, et al. Topical review: A survey of signal processing algorithms in brain-computer interfaces based on electrical brain signals. J Neural Eng. 2007;4(2):R32-R57.

11. Vidal JJ. Real-time detection of brain events in EEG. Proceedings of the IEEE. 1977;65(5):633-664.

12. Leuthardt EC. A brain-computer interface using electrocorticographic signals in humans. J Neural Eng. 2007;1(2):63-71.

13. Cincotti F, Mattia D, Aloise F, et al. High-resolution EEG techniques for brain-computer interface applications. J Neurosci Methods. 2007; 167(1):31-42.

14. Becedas J. Brain-machine interfaces: basis and advances. IEEE Transactions On Systems, Man, And Cybernetics. 2012;42(6):825836.

15. Nicolelis MAL. Actions from thoughts. NATURE. 2001;409(6818):403-407.

16. Nicolas-Alonso LF, Gomez-Gil J. Brain computer interfaces, a review. Sensors. 2012;12(2):1211-1279.

17. Bogue R. Brain-computer interfaces: control by thought. Industrial Robot: An International Journal. 2010;37(2):126-132.

18. Schalk G, Leuthardt EC. Brain-computer interfaces using electrocorticographic signals. IEEE Rev Biomed Eng. 2011:4:140154.
19. Maruyama T, Makikawa M, Shiozawa N, et al. ECG measurement using capacitive coupling electrodes for man-machine emotional communication. IEEE/ICME International Conference on Complex Medical Engineering; 2007;378-383.

20. Basics of surface electromyography applied to psychophysiology. Thought Technology Ltd; 2008.

21. Ferraioli F, Formisano A, Martone R, et al. Criteria for the optimal design of magneto-encephalography measurement system. IEEE Transactions On Magnetics. 2006;42(4):1155-1158.

22. Weiskopf N, Mathiak K, Bock SW, et al. Principles of a brain-computer interface (bci) based on real-time functional magnetic resonance imaging (fmri). IEEE Trans Biomed Eng. 2004;51(6):966-970.

23. Yanagisawa K, Sawai H, Tsunashima H. Development Of Nirs-Bci System Using Perceptron. 12th International Conference On Control, Automation And Systems (Iccas); 2012 Oct 17-21; JeJu Island, South Korea. 2012. p. 1531-1535.

24. Rao R, Scherer R. Brain-computer interfacing [In The Spotlight]. IEEE Signal Process Mag. 2010;27(4):150-152.

25. Chen YY, Lai HY, Lin SH, et al. Design and fabrication of a polyimide-based microelectrode array: application in neural recording and repeatable electrolytic lesion in rat brain. J Neurosci Methods. 2009; 182(1):6-16.

26. Yamakawa T, Yamakawa T, Inoue T. Minimally invasive ecog recording using the novel subdural electrodes manipulated by a shape memory alloy guidewire. Epilepsia. 2011;52:201-201.

27. Belluomo M, Bucolo L, Fortuna, et al. Robot control through braincomputer interface for pattern generation. AIP Conference Proceedings. 2012;1389(1):243-251.

28. Kanehiro F, Mizcuchi I, Koyasako K, et al. Development of a remotebrained humanoid for research on whole body action. International IEEE Conference on Robotics \& Automation; Ixuven, Belgium. 1998:1302-1307.

29. Moxon KA. Brain-control interfaces for sensory and motor prosthetic devices. IEEE; 2001:3445-3448, 2001.

30. Kim HK, Biggs SJ, Schloerb DW, et al. Continuous shared control for stabilizing reaching and grasping with brain-machine interfaces. IEEE Trans Biomed Eng. 2006;53(6):1164-1173.

31. Chella A, Pagello E, Menegatti E, et al. A BCI tele operated museum robotic guide. International IEEE Conference on Complex, Intelligent and Software Intensive Systems; 2009:783-788.

32. Yoshioka M, Zhu C, Yoshikawa Y, et al. Construction of real-time BMI control system based on motor imagery. International IEEE Conference on Robotics and Biomimetics, Thiland; 2011. p. 198-203.

33. Long J, Li Y, Wang H, et al. A hybrid brain computer interface to control the direction and speed of a simulated or real wheelchair. IEEE Trans Neural Syst Rehabil Eng. 2011;20(5):720-729.

34. Pfurtscheller G, Neuper C, Müller GR, et al. Graz-BCI: State of the Art and Clinical Applications. IEEE Trans Neural Syst Rehabil Eng. 2003;11(2):177-180. 